Citation: dasNair, R., Kontou, E., Smale, K., Barker, A., \& Lincoln, N.B. (2015). Comparing individual and group intervention for psychological adjustment in people with multiple sclerosis: A feasibility randomised controlled trial. Clinical Rehabilitation. DOI: 10.1177/0269215515616446

\title{
Comparing individual and group intervention for psychological adjustment in people with multiple sclerosis: A feasibility randomised controlled trial
}

\section{Roshan das Nair,', Eirini Kontou', Kathryn Smale', Alex Barker' and Nadina B Lincoln'}

\begin{abstract}
Objective: To modify a published group intervention for adjustment to multiple sclerosis (MS) to suit an individual format, and to assess the feasibility of a randomised controlled trial (RCT) to compare individual and group intervention for people with multiple sclerosis and low mood.

Design: Feasibility randomised controlled trial.

Setting: Participants were recruited through healthcare professionals at a hospital-based multiple sclerosis service and the MS Society.

Subjects: People with multiple sclerosis.

Interventions: Adjustment to multiple sclerosis in individual or group delivery format.

Main measures: Participants completed mood and quality of life assessments at baseline and at four-month follow-up. Measures of feasibility included: recruitment rate, acceptability of randomisation and the intervention (content and format), and whether the intervention could be adapted for individual delivery. Participants were screened for inclusion using the General Health Questionnaire-12 and Hospital Anxiety and Depression Scale, and were randomly allocated to receive either individual or group intervention, with the same content.

Results: Twenty-one participants were recruited (mean age 48.5 years, SD 10.5) and were randomly allocated to individual $(n=11)$ or group $(n=10)$ intervention. Of those offered individual treatment, nine $(82 \%)$ completed all six sessions. Of those allocated to group intervention, two $(20 \%)$ attended all six sessions and three (30\%) attended five sessions. There were no statistically significant differences between the groups on the outcome measures of mood and quality of life.
\end{abstract}

1 Division of Rehabilitation \& Ageing, University of Nottingham, UK

2 Department of Clinical Psychology and Neuropsychology,

Nottingham University Hospitals NHS Trust, UK
Corresponding author:

Roshan das Nair, Division of Rehabilitation and Ageing,

Queens Medical Centre, B98, B floor, Nottingham, NG7

1UH, UK.

E-mail: roshan.nair@nottingham.ac.uk 
Conclusions: The intervention could be provided on an individual basis and the trial design was feasible. There were lower attendance rates at group sessions compared to individual sessions.

\section{Keywords}

Multiple Sclerosis, randomized controlled trial, adjustment, cognitive behavioural therapy

Received: 5 February 2015; accepted: 16 October 2015

\section{Introduction}

Cognitive behavioural therapy is an established psychological treatment for mood disorders, with an extensive evidence base, and has been found to facilitate the adjustment process for people diagnosed with multiple sclerosis. ${ }^{1}$ A review of the effectiveness of cognitive behavioural therapy for people with multiple sclerosis ${ }^{2}$ identified seven randomised controlled trials (RCTs) comparing cognitive behavioural therapy with a control condition. These included evaluations of cognitive behavioural therapy using individual format $(n=3)$, group format $(n=3)$, and computerised format $(n=1)$. Metaanalysis revealed a significant positive effect of cognitive behavioural therapy on depression for people with multiple sclerosis. An indirect comparison of individual and group intervention showed no significant differences in treatment outcomes. A further recent evaluation of combined face-to-face and telephone cognitive behavioural therapy supported these findings, ${ }^{3}$ especially for those with moderate levels of distress.

Another review of psychological interventions for people with multiple sclerosis focused on the format of intervention, i.e. group interventions, rather than interventions based on a specific psychological model. ${ }^{4}$ This review of 14 studies concluded that group psychological interventions were less effective than individual cognitive behavioural therapy or medication for depression in the short term but had comparable long-term outcomes. ${ }^{4}$ However, a meta-analysis was not conducted.

Some studies have suggested that it is the group context, in which there is interaction with other people that is of primary importance, ${ }^{5}$ but none have provided a direct comparison of individual and group interventions with similar content. Forman and Lincoln's ${ }^{6}$ pilot RCT evaluating a sixsession adjustment group intervention based on cognitive behavioural therapy principles for people with multiple sclerosis and low mood, showed a significant positive effect on depressive symptoms. Lincoln et al. ${ }^{7}$ further supported the results of this pilot study and showed that this adjustment group improved mood and reduced the impact of multiple sclerosis on daily life. It was also shown to be costeffective. $^{8}$

Therefore, there is no clear consensus of the relative merits and limitations of both formats of this intervention. The aim of our study was to assess the feasibility of an RCT to compare the format of an adjustment group based on cognitive behavioural therapy principles, 6,7 and to compare the effect of the format of delivery of the intervention (individual or group) on mood and quality of life.

\section{Method}

The study was a single centre, feasibility randomised controlled trial comparing a group psychological adjustment intervention based on cognitive behavioural therapy principles with the same intervention delivered on an individual basis. Ethical approval was granted by Nottingham Research Ethics Committee 1.

Participants were recruited through the National Health Service (NHS) and MS Society. Clinicians working in NHS Multiple Sclerosis Clinics in Nottingham invited people with multiple sclerosis to take part. Patients were given information about the study, including a participant information sheet 
with a reply slip to return their contact details to the researchers if they were interested in taking part. Potential participants were contacted by the researchers and were sent a screening pack, containing two mood measures, the General Health Questionnaire-12 (GHQ-12) ${ }^{9}$ and the Hospital Anxiety and Depression Scale (HADS), ${ }^{10}$ to complete and return by post. Study posters were displayed on the MS Society website and in their local branch newsletters. Those who were recruited through the MS Society were also sent a screening pack to return by post.

Participants were included if they had a diagnosis of multiple sclerosis and scored three or more out of 12 on the GHQ-12 or eight or more out of 21 on the HADS Anxiety or Depression subscales.

Participants who were eligible for inclusion were contacted by telephone and an appointment was made to further discuss the study and to complete a consent form. Baseline assessments were administered in a single one-hour session at the university or at participants' homes by a clinical psychologist or a trained research psychologist. Participants were excluded if they did not speak English and if they were unable to attend group sessions (if they were to be allocated to group treatment).

Those who met the criteria and who gave informed consent were included. Personal details, demographic characteristics, type and duration of multiple sclerosis were recorded. Participants then completed the following baseline measures: (i) Guy's Neurological Disability Scale (GNDS), ${ }^{11}$ a 12-item scale that assesses impairments due to multiple sclerosis in the following areas: cognition, mood, vision, communication, swallowing, upper limb, lower limb function, bladder, bowel, sexual, fatigue, and 'other' problems. The range of possible scores is between 0 and 60 , with higher scores indicating greater levels of disability. (ii) Beck Depression Inventory-II (BDI-II), ${ }^{12}$ a 21 -item measure of severity of depressive symptoms, which has been used in previous trials of psychological intervention for people with multiple sclerosis. Higher scores indicate greater depression. (iii) Multiple Sclerosis Impact Scale-29 (MSIS), ${ }^{13}$ a disease-specific quality of life scale with good psychometric properties. It comprises 20 items on physical impact of multiple sclerosis and nine items on psychological impact of multiple sclerosis. Higher scores indicate a greater impact of multiple sclerosis on quality life. (iv) Multiple Sclerosis Self-efficacy Scale (MSSE), ${ }^{14}$ a14-item scale to assess the extent to which participants felt in control of their condition. Higher scores indicate greater self-efficacy.

Participants were asked whether they would prefer individual or group treatment, but were informed that the allocation would be random. This was to ascertain preference and to determine whether dropouts were related to not receiving the preferred treatment. Participants were randomly allocated to either individual or group treatment (1:1 ratio). Randomisation was conducted using a web-based random number generator by an independent researcher who was not involved in the assessment or intervention. The group treatment programme was offered to 3-8 participants who were all able to attend the specified location on the same dates. Individual treatment was arranged at times to suit each participant and conducted in participants' homes or at the university.

Treatment sessions were delivered by a clinical psychologist or a trained research psychologist. Both received supervision from experienced researchers with a background in clinical psychology and working with people with multiple sclerosis. Both treatments were conducted in accordance with the written, structured group manual developed by Forman and Lincoln. ${ }^{6}$ Adaptations were made to the group manual handouts and worksheets, as required, to suit the individual format. Both treatments were designed to be delivered in six sessions, although the content of the individual treatment could be delivered flexibly across sessions (i.e., therapists could complete content in one or more sessions, depending on the needs of the participants). Group sessions were video recorded to monitor the fidelity of the intervention. Videos allowed us to determine who was saying what, which audio recordings alone would not have allowed us to do easily. This was not a problem for individual sessions (where there were only two speakers), so audio recordings were used instead. 
At four months after random allocation, participants were sent a booklet of outcome questionnaires to complete and return by post using a prepaid reply envelope. The measures included GHQ$12,{ }^{9}$ HADS,${ }^{10}$ BDI-II, ${ }^{12}$ MSIS $^{13}$ and MSSE. ${ }^{14}$ These assessments were used because they tapped into the outcomes of interest for this study (mood and quality of life). In addition to this, we included a measure of self-efficacy (i.e., perceived capability or control), because treatment may improve selfefficacy and it is believed to influence both mood and quality of life in people with multiple sclerosis. ${ }^{15}$ Outcome data were entered onto a database by a researcher blind to group allocation. Analyses were conducted using SPSS version 21.

\section{Results}

The flow of participants through the study is shown in Figure 1.

We had an $80 \%(n=27)$ response rate for return of screening questionnaires, and $62 \%(n=21)$ of potential participants met the inclusion criteria.

The demographic and baseline characteristics of participants are shown in Table 1. One participant (shown in the table as an unknown type of multiple sclerosis) was later found to have a diagnosis of neuromyelitis optica, but was retained in the study.

Prior to randomisation, participants were asked to state their treatment format preference to determine whether participants had a strong preference which if not met could have influenced their decision to continue with the trial, but reminded that allocation would be random. Seven participants did not report a treatment preference, seven preferred to be allocated to group treatment and seven to individual treatment. Of the four participants who did not attend for treatment, three were not allocated to their preferred treatment.

Of the 11 allocated to individual treatment, we were unable to contact one participant who therefore received no treatment. Of the 10 allocated to group treatment, seven participants were treated in one group and three in another. The reasons for non-attendance at any group sessions were bereavement, a relapse, and not being offered their preferred treatment format.
Individual sessions lasted approximately one hour, and group sessions lasted approximately two hours.

Outcome questionnaires were returned by 16 (76\%) of the participants, eight $(73 \%)$ allocated to individual treatment and eight $(80 \%)$ allocated to group treatment. The distributions of scores on the outcome assessments are shown in Table 2.

The groups were comparable in demographic characteristics at baseline. The data were not normally distributed, and the sample size was small, so non-parametric statistics were used.

The two groups were compared on the outcome measures using Mann Whitney U tests. There were no statistically significant differences between the groups $(P=0.07$ to $P=0.57$ ), but on all outcome measures the scores were better for individual than group treatment. The effect sizes ranged from 0.29 (BDI-II) to 0.86 (GHQ-12). These suggest that there is a considerable benefit of individual therapy over group therapy.

Based on the effect size for the MSIS Psychological subscale (0.40), we conducted a sample size calculation for a definitive trial. With $80 \%$ power and an alpha of 0.05 , for a two-tailed test, a sample size of 96 per group will be needed.

\section{Discussion}

The study design was feasible and acceptable to people with multiple sclerosis. The manualised individual treatment was feasible, had better attendance rates and appeared to have better outcomes compared to the group format, which is consistent with previous meta-analyses. ${ }^{4}$

Recruitment was slower than expected but achievable. Based on our previous experience, we had anticipated recruiting approximately six participants per month, but in reality this was closer to three per month. As $21 \%$ of those invited did not return the screening questionnaires, a definitive study would benefit from sending reminder letters or where possible, a single phone call to remind potential participants. Also, providing the option for participants to consent and complete the screening questionnaires at their routine clinic visits may improve recruitment and response rates. 


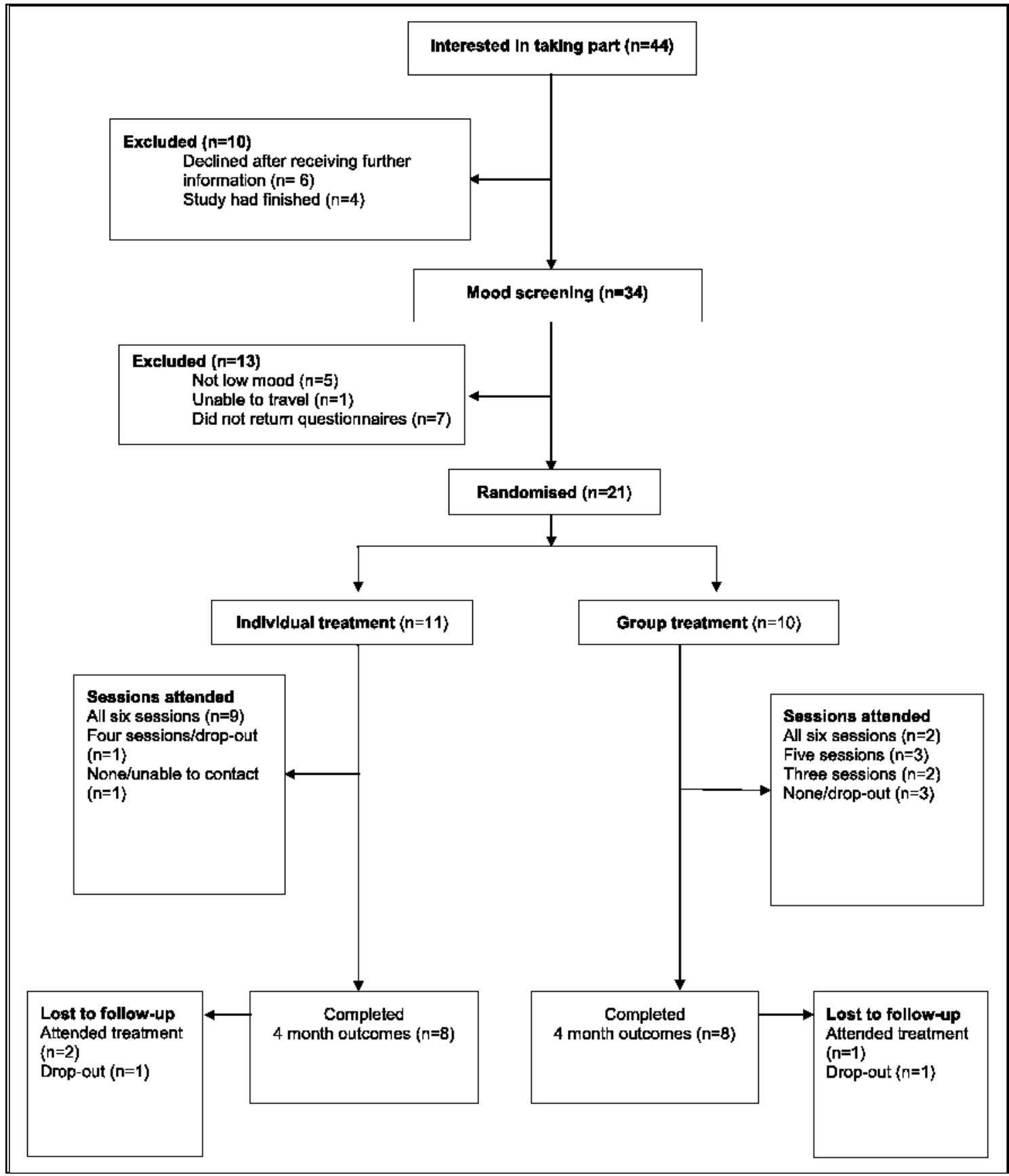

Figure 1. Trial flowchart.

One participant did not attend treatment because of not being allocated to their preferred treatment, so for most participants $(95 \%)$ randomisation was acceptable. Outcomes were completed by $76 \%$, and suggest that postal or telephone reminders or face-to-face follow-up assessments would be 
Table 1. Baseline demographic and clinical characteristics.

\begin{tabular}{|c|c|c|c|c|}
\hline \multirow[t]{2}{*}{ Characteristics } & \multicolumn{2}{|c|}{$\begin{array}{l}\text { Individual treatment } \\
n=11\end{array}$} & \multicolumn{2}{|c|}{$\begin{array}{l}\text { Group treatment } \\
n=10\end{array}$} \\
\hline & $n$ & $\%$ & $n$ & $\%$ \\
\hline \multicolumn{5}{|l|}{ Gender } \\
\hline Men & 3 & 27 & 3 & 30 \\
\hline Women & 8 & 73 & 7 & 70 \\
\hline \multicolumn{5}{|l|}{ Marital status } \\
\hline Single & 2 & 18 & 5 & 50 \\
\hline Married/partnered & 8 & 73 & 5 & 50 \\
\hline Widowed/divorced/separated & 1 & 9 & 0 & 0 \\
\hline \multicolumn{5}{|l|}{ Living arrangements } \\
\hline Alone & 2 & 18 & 4 & 40 \\
\hline With family/partner & 9 & 82 & 6 & 60 \\
\hline \multicolumn{5}{|l|}{ Ethnicity } \\
\hline White British & 11 & 100 & 7 & 70 \\
\hline Other & 0 & 0 & 3 & 30 \\
\hline \multicolumn{5}{|l|}{ Type of multiple sclerosis } \\
\hline Relapsing remitting & 9 & 82 & 5 & 50 \\
\hline Progressive & 1 & 9 & 3 & 30 \\
\hline Benign & 1 & 9 & 0 & 0 \\
\hline Not known & 0 & 0 & 2 & 20 \\
\hline Currently receiving MS disease modifying medication & 8 & 73 & 6 & 60 \\
\hline Currently receiving antidepressant medication & 3 & 27 & 4 & 40 \\
\hline Other medical conditions & 3 & 27 & 6 & 60 \\
\hline \multicolumn{5}{|l|}{ Recruitment source } \\
\hline MS Society newsletter & 6 & 55 & 8 & 80 \\
\hline Hospital & 5 & 45 & 2 & 20 \\
\hline \multicolumn{5}{|l|}{ Treatment preference } \\
\hline Individual & 3 & 27 & 4 & 40 \\
\hline Group & 4 & 36 & 3 & 30 \\
\hline Either & 4 & 36 & 3 & 30 \\
\hline \multicolumn{5}{|l|}{ Allocated to treatment preference } \\
\hline Yes & 3 & 27 & 3 & 30 \\
\hline No & 4 & 36 & 4 & 40 \\
\hline \multirow[t]{2}{*}{ Indifferent } & 4 & 36 & 3 & 30 \\
\hline & Mean & SD & Mean & SD \\
\hline Age (years) & 48.9 & 10.4 & 48 & 11.2 \\
\hline Time since diagnosis & 9.3 & 6.8 & 8.9 & 6.4 \\
\hline Guys Neurological Disability Scale & 20.6 & 7.8 & 22.3 & 8.2 \\
\hline
\end{tabular}

$n=$ sample size; $S D=$ standard deviation.

needed in a definitive trial to improve completion rates.

The acceptability of the content of the intervention was established by Forman and Lincoln, ${ }^{6}$ but we wanted to know whether this was acceptable for delivery in one-to-one sessions. The individual treatment was well received by participants and adherence was very good, with $88 \%$ of sessions 


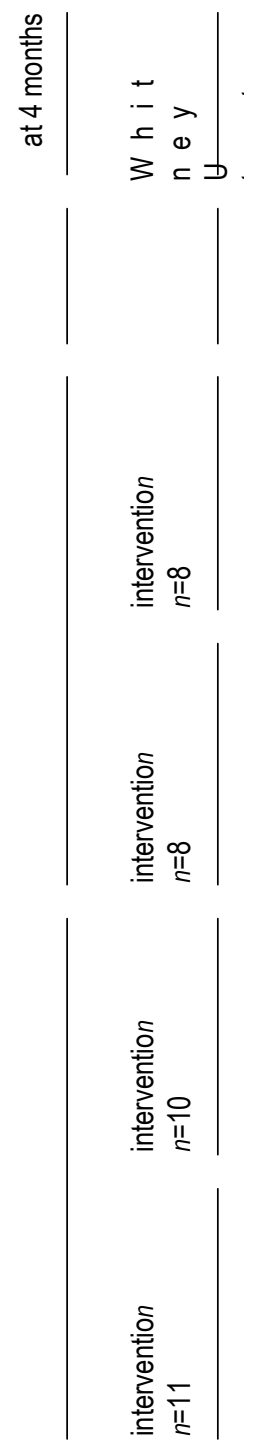

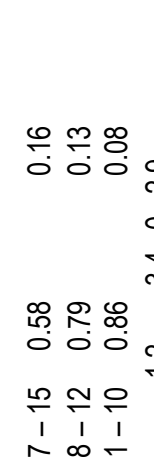

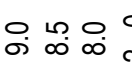

$0, \infty$
$c$

으 $\infty \mathrm{m}$

c

c

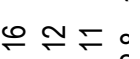

$\begin{array}{lll}1 & 1\end{array}$

c

웅요

으응 ${ }^{\infty}$

m는

l 11

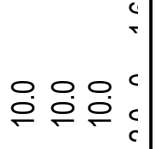

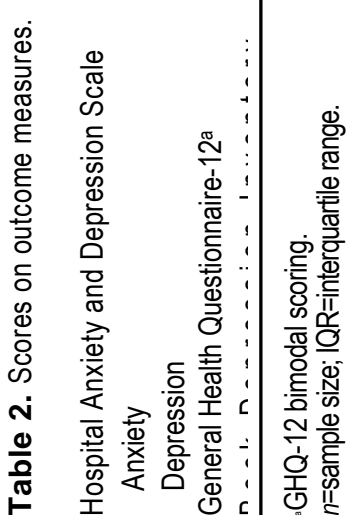


attended. Adherence in the group intervention was lower, with $55 \%$ of sessions attended.

Attendance at group sessions has been found to be a problem previously and the proportion attending was consistent with that found in previous research. ${ }^{16}$ One hypothesis is that the success of the groups is dependent on group cohesion, which is often linked to shared identities, ideologies, and interests. While all participants had low mood and multiple sclerosis, this in itself may not have been sufficient to foster cohesion. For the Holmes et al. ${ }^{16}$ study, non-attendance was more common among men than women. They were in the minority in each group and may not have been able to connect to the others. Another hypothesis is that people may feel more obliged to attend individual rather than group sessions, because the focus of therapy is solely on them and not on others in the former. Yet another hypothesis is that people may have felt they were getting more out of the individual sessions than the group sessions, which may reflect the differences in the outcomes between the different delivery formats (considered below). Furthermore, individual sessions allowed for more focus on certain topics considered relevant for each individual. For instance, if the participant was very anxious, the focus was more on the worry sessions than others. If a topic was not as relevant, then this was only covered briefly. Also, individual sessions could be rearranged in the event of participant illness or other commitments, whereas it was difficult to reschedule group sessions at short notice. The reason for one participant not attending group sessions was difficulty with travel and this did not occur in those allocated to individual therapy, as treatment sessions could be conducted in participants' homes.

In terms of the completeness of outcome data, five $(24 \%)$ were lost to follow-up and there was complete data on 16 participants $(76 \%)$ at the 4 month outcome. The loss to follow-up was not related to attendance at treatment sessions. Indeed, only one had not attended any sessions, and two had attended all sessions. In a definitive study, with longer follow-up periods, it may be beneficial to have regular phone or email contact with all participants to ensure that they remain interested in the study. More flexible means of collecting these outcomes should also be considered, such as online surveys or mobile phone apps, or even by collecting the data over the phone. These strategies may improve quality and amount of outcome data. However, direct contact with participants raises the risk of the outcome assessor becoming unblinded.

Scores on mood measures were better for individual treatment than group treatment at the 4-month outcome assessment, but these differences were not statistically significant. However, they are clinically important differences which suggest there may be advantages of individual treatment. The lack of statistically significant results was influenced by the small sample size. The effect sizes varied between 0.29 and 0.86 but five of the seven effect sizes calculated were greater than 0.5 , which indicated medium effect and suggests that with a larger sample differences in outcome may be detected. Based on this, we calculated the parameter estimates for a fully powered study, and determined that a sample size of 96 per group would be needed for a definitive trial. Imbalance between groups in terms of type of multiple sclerosis, and the small sample size meant that the effect of type of multiple sclerosis on outcomes could not be determined.

No serious adverse events were reported during treatment, but two participants who received individual treatment continued to report suicidal thoughts but with no intent to carry them out, which related to their response on the baseline Beck Depression Inventory score on the 'suicidal thoughts' item. Both cases were assessed by a clinical psychologist and neither was deemed to be at risk of suicide, but for one participant, it was agreed that the research team would pass on this information to their General Practitioner. A recent systematic review found higher suicide rate in people with multiple sclerosis compared to the general population. ${ }^{17}$ Suicide risk, therefore, needs to be monitored carefully in future studies.

The study design was feasible and acceptable to people with multiple sclerosis. The manualised individual treatment was feasible and seemed to be beneficial, but needs to be tested in an adequately powered RCT. Furthermore, the cost-effectiveness 
of individual and group treatments needs to be ascertained.

We advocate that clinicians consider a mixed individual and group intervention, with individual treatment available for those who do not wish to attend groups. In such a mixed format, it may be possible to harness the strengths of both individual (e.g., individualised treatment, person-centred care, therapeutic alliance, better adherence, space to discuss some aspects in private) and group (e.g., therapeutic effects of the group, possibilities for socialisation and identity formation, sharing of information) delivery formats. This mixed format mimics some clinical interventions, but the clinical and cost-effectiveness of this specific intervention for people with multiple sclerosis is yet to be evaluated.

\section{Clinical Messages}

- More patients with multiple sclerosis offered psychologically-based adjustment therapy attended individual rather than group sessions.

- The study found a trend towards better outcomes after individual treatment; it was not statistically significant.

- A randomised controlled study including about 200 patients would be needed to establish the actual relative effectiveness.

\section{Acknowledgements}

We would like to thank Prof Cris Constantinescu, Dr Nikos Evangelou, and the MS Society for assistance with recruitment and the participants for giving up their time.

\section{Declaration of Conflicting Interests}

The authors declared no potential conflicts of interest with respect to the research, authorship, and/or publication of this article.

\section{Funding}

The authors disclosed receipt of the following financial support for the research, authorship, and/or publication of this article: This study was supported by a grant from the Nottingham Hospitals Charity.

\section{References}

1. Thomas PW, Thomas S, Hillier C, et al. Psychological interventions for multiple sclerosis. Cochrane Database of Systematic Reviews 2009; (1)CD004431.

2. Hind D, Cotter J, Thake A, et al. Cognitive behavioural therapy for the treatment of depression in people with multiple sclerosis: a systematic review and meta-analysis. BMC Psychiatry 2014; 14: 5.

3. Moss-Morris R, Dennison L, Landau S, et al. A randomized controlled trial of cognitive behavioral therapy (CBT) for adjusting to multiple sclerosis (the saMS trial): does CBT work and for whom does it work? J Consult Clin Psychol. 2013; 81: 251-262.

4. Firth N. Effectiveness of psychologically focused group interventions for multiple sclerosis: A review of the experimental literature. J Health Psychol 2014; 19: 789-801.

5. Rigby SA, Thornton EW and Young CA. A randomized group intervention trial to enhance mood and self efficacy in people with multiple sclerosis. Br J Health Psychol 2008; 13: 619-631.

6. Forman AC and Lincoln NB. Evaluation of an adjustment group for people with multiple sclerosis: a pilot randomized controlled trial. Clin Rehabil 2010; 24: 211-221.

7. Lincoln NB, Yuill F, Holmes J, et al. Evaluation of an adjustment group for people with multiple sclerosis and low mood: a randomised controlled trial. Mult Scler 2011; 17: 1250-1257.

8. Humphreys I, Drummond AER, Phillips C, et al. Cost-effectiveness of an adjustment group for people with multiple sclerosis and low mood: a randomized trial. Clin Rehabil 2013; 27; 963-971

9. Goldberg D. General Health Questionnaire (GHQ12). Windsor, UK: NFER-Nelson, 1992.

10. Zigmond AS and Snaith RP. The Hospital Anxiety and Depression Scale. Acta Psychiatr Scand 1983; 67: 361-370.

11. Sharrack B and Hughes RAC. The Guy's Neurological Disability Scale (GNDS): a new disability measure for multiple sclerosis. Mult Scler 1999; 5: 223-233.

12. Beck AT, Steer RA and Brown GK. Beck Depression Inventory-II (BDI-II). San Antonio, TX: Psychological Corporation, 1996.

13. Hobart J, Lamping D, Fitzpatrick R, et al. The Multiple Sclerosis Impact Scale (MSIS-29): a new patient-based outcome measure. Brain 2001; 124: 962-973.

14. Domenech CL, Thornton EW, Young CA, et al. Development of a self efficacy scale for people with multiple sclerosis. Mult Scler 1999; 5: S144.

15. Barnwell AM and Kavanagh DJ. Prediction of psychological adjustment to multiple sclerosis. Soc Sci Med 1997; 45: 411-418.

16. Holmes JM, Ford E, Yuill F, et al. Attendance at a Psychological Support Group for People with Multiple Sclerosis. Disabil Rehabil 2012; 34: 1323-1327.

17. Pompili ML, Forte A, Palermo M, Stefani H, Lamis DA, Serafini G, et al. Suicide risk in multiple sclerosis: a systematic review of current literature. J Psychosom Res 2012; 73:411-417. 